\section{(6) OPEN ACCESS}

\title{
Optimising deep anterior lamellar keratoplasty (DALK) using intraoperative online optical coherence tomography (iOCT)
}

\author{
Philipp Steven, ${ }^{1}$ Carolin Le Blanc, ${ }^{1}$ Eva Lankenau, ${ }^{2}$ Marc Krug, ${ }^{2}$ Stefan Oelckers, ${ }^{3}$ \\ Ludwig M Heindl, ${ }^{1}$ Uta Gehlsen, ${ }^{1}$ Gereon Huettmann, ${ }^{4}$ Claus Cursiefen ${ }^{1}$
}

\begin{abstract}
${ }^{1}$ Department of Ophthalmology, University of Cologne, Cologne, Germany

${ }^{2}$ OptoMedical Technologies $\mathrm{GmbH}$, Luebeck, Germany ${ }^{3}$ Moeller-Wedel GmbH, Wedel, Germany

${ }^{4}$ Institute of Biomedical Optics, University of Luebeck, Luebeck, Germany
\end{abstract}

* Correspondence to Dr Philipp Steven, Department of Ophthalmology, University Hospital of Cologne, Kerpener Strasse 62, Cologne 50937, Germany;

philipp.steven@uk-koeln.de

Received 5 November 2013 Revised 6 January 2014 Accepted 19 January 2014 Published Online First 3 March 2014

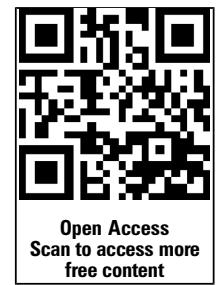

\section{ABSTRACT}

Background/aims To describe the use of intraoperative online optical coherence tomography (iOCT) for improving deep anterior lamellar keratoplasty (DALK) surgery.

Methods Retrospective case series of 6 eyes of 6 male patients with keratokonus, corneal dystrophy or herpetic stromal scars undergoing DALK were investigated using intraoperative optical coherence tomography and postsurgical image/video analysis. Main outcome measures were: visibility of surgical steps, especially, assessment of placement depth of injection needle, preparation of bare Descemet's membrane and drainage of interface fluid.

Results $\mathrm{IOCT}$ enables real-time visualisation of all surgical steps of DALK procedure in all patients. Placement of air injection needle above Descemet's membrane was reliably monitored as was presence of bare Descemet's membrane and potential interface fluid. Conclusions $\mathrm{IOCT}$ assists with visualisation of injection needle placement and with assessment of bare Descemet's membrane as well as interface fluid during the DALK procedure. Overall iOCT may be a helpful device that supports surgeons in all steps of DALK procedure.

\section{INTRODUCTION}

Deep anterior lamellar keratoplasty (DALK) using the big-bubble technique was first described by Anwar and Teichmann. ${ }^{1}$ DALK uses a stromal airinjection for dissection of stromal tissue from Descemet's membrane (DM) followed by engraftment of a stromal donor button, and is performed for treatment of corneal stromal diseases such as keratokonus, hereditary corneal degeneration or corneal scarring. This technique has demonstrated advantages over perforating keratoplasty (PKP), such as absent endothelial transplant rejections ${ }^{2-4}$ and reduced postoperative complications. The most important limiting factor for visual outcome in DALK is incomplete stromal dissection. ${ }^{5}$ Nonetheless, the American Academy of Ophthalmology in a recent technology assessment report suggests DALK to be as good as PKP in terms of visual acuity, but better in terms of safety because of absent risk of endothelial immune reactions. ${ }^{6}$

Adaption of DALK worldwide is slow, partly because of the technical challenges of this surgery and its non-standardised nature. That is partly related to the limited visualisation of key surgical steps during surgery which is based on tissue transparency and hindered estimation of depth ratio due to en-face view onto the cornea through the operation microscope. Therefore, optical cross-sections would allow the surgeon to better navigate and control the procedure, especially crucial steps, such as assessment of depth of intrastromal needle placement, achievement of pure DM preparation and absence of interface fluid at the end of surgery.

Recently, optical coherence tomography (OCT) has been modified to be used in an intraoperative setting. ${ }^{7}$ Hereby, two main approaches are possible: (1) using hand-held devices ${ }^{89}$ or (2) integration of the OCT into the operation microscope. ${ }^{10}$ The latter has several advantages, such as online visualisation of all surgical steps without the necessity of interrupting the procedure and alignment of the OCT-image to any given zoom and focus-step of the microscope. This study is the first to evaluate intraoperative OCT (iOCT) technology to visualise DALK surgery online without the necessity of discontinuing the procedure for OCT measurements.

\section{METHODS}

\section{Intraoperative online OCT technology}

For intraoperative online OCT evaluation, a microscope-mounted, commercially available spectral-domain OCT camera (iOCT, OptoMedical Technologies GmbH, Luebeck, Germany) using a $840 \mathrm{~nm}$ central wavelength and performing $10000 \mathrm{~A}$-scans/s was used. The iOCT was connected to the camera port of an OCT-compatible MOELLER Hi-R 900 A near infrared microscope (Moeller-Wedel, Wedel, Germany). ${ }^{11}$ The OCT image was displayed on a separate touch screen in front of the surgeon's visual field to enable easy exchange between microscopic and OCT images. iOCT imaging included recording of highresolution videos and images of approximately $10 \mu \mathrm{m}$ axial resolution in air. Image size was $4.2 \mathrm{~mm}$ axially in air, respectively, $3.2 \mathrm{~mm}$ axially in water, and between 5 and $29 \mathrm{~mm}$ in lateral direction, depending on the microscopic zoom factor used.

\section{Patients}

iOCT was used in six consecutive male patients aged 25-64 years, suffering from keratokonus (3 cases), stromal scarring ( 2 cases) or hereditary corneal stromal dystrophy Francois (1 case) (table 1).

In two of the cases, DALK had to be converted to penetrating keratoplasty due to larger DM perforation during lamellar preparation.

Evaluation parameters included iOCT-visualisation of trephination depth, deep stromal placement of air 
Table 1 Patients, diagnosis 1 and procedure

\begin{tabular}{|c|c|c|}
\hline Patient & Diagnosis & Procedure/performance \\
\hline 1. Male, age 25 & Keratoconus & Pre-Descemet air bubbles, DM rupture, conversion into PKP \\
\hline 2. Male, age 32 & Keratoconus & Big bubble, DALK \\
\hline 3. Male, age 39 & Keratoconus & Pre-Descemet air bubbles, DM rupture, conversion into PKP \\
\hline 4. Male, age 38 & Corneal dystrophy Francois & Big bubble, DALK \\
\hline 5. Male, age 64 & Herpetic stromal scarring & Pre-Descemet air bubbles, microbubble incision technique, DALK \\
\hline 6. Male, age 49 & Herpetic stromal scarring & Pre-Descemet air bubbles, microbubble incision technique, DALK \\
\hline
\end{tabular}

injection needle, preparation of bare DM, interface fluid and interface alignment of the graft.

\section{Procedure}

DALK was performed as described previously ${ }^{12}{ }^{13}$ Briefly, the recipient cornea was trephined to $90 \%$ of minimal corneal thickness at the $8 \mathrm{~mm}$ paracentral region using a Hessberg-Barron trephine (Domilens, Hamburg) of $7.75 \mathrm{~mm}$. Thickness was preoperatively assessed using Pentacam. A 30 -gauge injection needle was placed in deep tissue directly above DM, and the placement of the needle tip was observed using intraoperative OCT. Then, air was injected until the border of the whitening expanded towards the trephination interface. Following lamellar dissection of the superficial and mid-stromal tissue a pre-Descemet large air cavity (big-bubble) was opened and the remaining tissue was dissected from DM to obtain a bare DM situation under viscoelastic protection (injected into the space). The donor tissue was placed endothelial side up into a Hanna punch block (Moria, Doylestown, Pennsylvania, USA) and DM was stripped from the cornea. DM grafts could then be used for Descemet membrane endothelial keratoplasty (DMEK) surgery in a split cornea transplantation. ${ }^{13}$ An $8 \mathrm{~mm}$ diameter graft was cut (using a Hessberg-Barron trephine) and transferred onto the recipient's cornea. The graft was fixated by 16 single sutures or two double running continuous sutures, and remaining interface fluid was drained by gentle massage of the corneal surface and opening of interface using a blunt spatula.

\section{Donor tissue}

Four grafts were organ-cultured in minimal essential medium (MEM) (Biochrom, Berlin, Germany) at $32^{\circ} \mathrm{C}$ and deswelled in MEM containing 5\% dextran (Biochrom, Berlin, Germany) at $32^{\circ} \mathrm{C}$.

Two grafts were organ-cultured in minimal essential medium (Cornea max R, Eurobio Laboratoires, Les Ulis, France) at $31^{\circ} \mathrm{C}$ and deswelled in minimal essential medium containing 5-6\% dextran (Cornea Jet R, Eurobio Laboratoires, Les Ulis, France).

No information was obtainable on exact postmortem time from the provider eye bank, however, due to legal requirements, all donor eyes were collected within $24 \mathrm{~h}$ postmortem. Donor age range from $18-72$ years, ( 4 male, 2 female), no previous eye diseases were reported.

\section{Retrospective image analysis}

For retrospective image analysis, and to conduct depth measurements, the corresponding pixel size of the $\mathrm{x}$-axis of the OCT image was set to 840 pixels, according to the axial optical window depth of $4.2 \mathrm{~mm}$. Therefore, the axial pixel size was set to $5 \mu \mathrm{m}$. To correct the index of refraction inside corneal tissue of 1.35 , the pixel size was divided by this factor resulting in a value of $3.7 \mu \mathrm{m}$. Using Image $\mathrm{J}$, the distances between the corneal epithelium, endothelium and the centre of the airinjection needle were measured, and the numbers of pixels converted to the true distance in $\mu \mathrm{m}$.

\section{RESULTS}

Intraoperative OCT enables monitoring of all surgical steps of DALK in all patients analysed (6/6: 100\%)

Trephination depth was accurately imaged (Figure 1A), and needle insertion could be reliably monitored in close proximity to DM (Figure 1B). Air injection into the posterior stroma was observed and resulted in whitish staining of the OCT image due to increased tissue scattering by air bubbles. ${ }^{14}$ In two out of six cases, a big bubble formation was visible (Figure 1C) in deep stromal location. Superficial and deep stromal tissue preparation (Figure 1D,E) was monitored reliably until bare DM was obtained (Figure 1F). Graft insertion and suturing was monitored, and interface congruence was controlled by intraoperative OCT (Figure 1G). These steps were reliably monitored in 6/6 patients, however, in two cases, rupture of DM during deep stromal preparation led to conversion into PKP. In these two cases, no initial big-bubble formation was achieved.

\section{iOCT guides in 'microbubble-incision technique'}

In $4 / 6$ cases no 'big-bubble formation' was observed, however, small air bubbles were evenly distributed throughout the whole corneal stroma (Figure 2A). Following superficial lamellar stromal dissection (Figure $2 \mathrm{~B}$ ) a $15^{\circ} \mathrm{knife}$ was used to incise individual air bubbles in order to form a larger cavity according to the previously described 'micro-bubble incision technique"15 (Figure 2C,D). This pre-Descemet cavity could then be opened (Figure 2E), and preparation of bare DM could be performed (Figure 2F). By this approach 2/4 cases could be 'rescued', and DALK was performed. In the remaining two patients, DM ruptured and PKP was performed.

\section{iOCT helps in assessment of interface fluid and interface steps at the end of surgery}

After placement of the graft into the corneal recipient's bed and suturing, remaining interface fluid could be detected by intraoperative OCT (Figure 3A) in two out of four patients. Following gentle massage of the corneal surface and blunt opening of interface in all four quadrants under air tamponade of anterior chamber, remaining fluid could safely be drained (Figure 3B).

Additionally, congruence of graft and donor interface could be secured, and interface steps corrected before end of the surgery. 

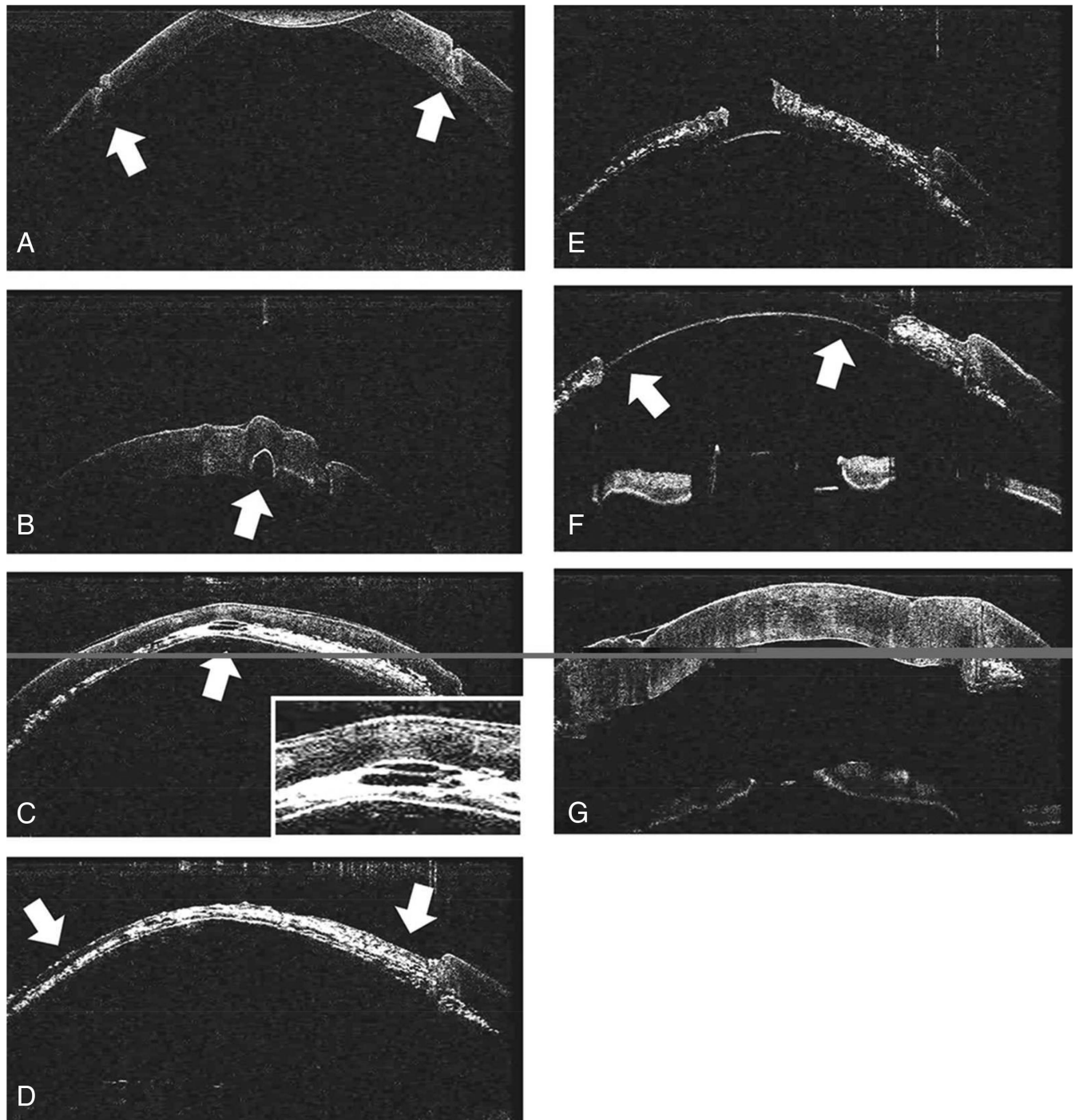

Figure 1 Intraoperative online optical coherence tomography enables real-time visualisation of all surgical steps of deep anterior lamellar keratoplasty surgery including graft trephination ( $A$; arrows show peripheral trephination), needle insertion (B; arrow marks needle) into deep stroma, air insufflation into deep stroma with big-bubble formation in this patient (C; inset shows small intrastromal air pocket), superficial lamellar preparation (D), deep lamellar preparation (E and F; arrows show isolated Descemet's membrane) down to isolated Descemet's membrane and graft attachment at the end of the procedure $(\mathrm{G})$.

\section{Analysis of trephination depth and placement of air injection needle}

Retrospective image analyses of representative OCT images demonstrate a trephination depth of approximately 270 or $198 \mu \mathrm{m}$, and a placement of the injection needle at 311 or $296 \mu \mathrm{m}$ (Figure 4). The thickness of the cornea was measured with 389 or $333 \mu \mathrm{m}$. Trephination depth, therefore, reached $69 \%$ or $67 \%$ of the total corneal thickness, respectively. The air injection needle was placed at a depth of $80 \%$ or $88 \%$ of total corneal thickness; however, this value represents the centre core of the injection needle.

\section{DISCUSSION}

DALK is an established surgical technique for treatment of stromal corneal diseases, such as keratoconus. Several preparation steps are challenging such as localisation of instruments within the stroma or estimation of remaining tissue thickness in relation to DM because they cannot be visualised precisely by conventional en-face microscopy. Approaches, such as the preoperative measurement of the stromal thickness using
Pentacam ${ }^{16}$ are feasible. However, intraoperative visualisation of the trephination depth and placement of the needle would be superior over preoperative measurements and would aid the surgeon during the procedure.

OCT has been used to image the anterior segment of the eye ${ }^{17}$ prior to and after deep anterior keratoplasty to plan surgical steps and to analyse the operative outcome..$^{18}$ By contrast, online intraoperative optical coherence tomography (iOCT), enables to visualise tissues and, to a certain extent, instruments in virtual cross-section of the region of interest, thereby allowing to precisely identify different tissue structures, tissue thickness, and so on, without interrupting the procedure and with much higher accuracy. The adaptation of OCT for intraoperative use, in particular, in lamellar keratoplasty is, therefore, of great interest and has recently been shown by our group and others. ${ }^{78} 1920$ Whereas most approaches use customised and modified OCT systems so far used in patients before or after surgery ${ }^{18}$ or slit beam imaging ${ }^{21}$ during surgery with the necessity to interrupt the procedure, this iOCT-system is the first device that was specifically developed to be mounted on a surgical microscope. This allows especially during delicate surgical 

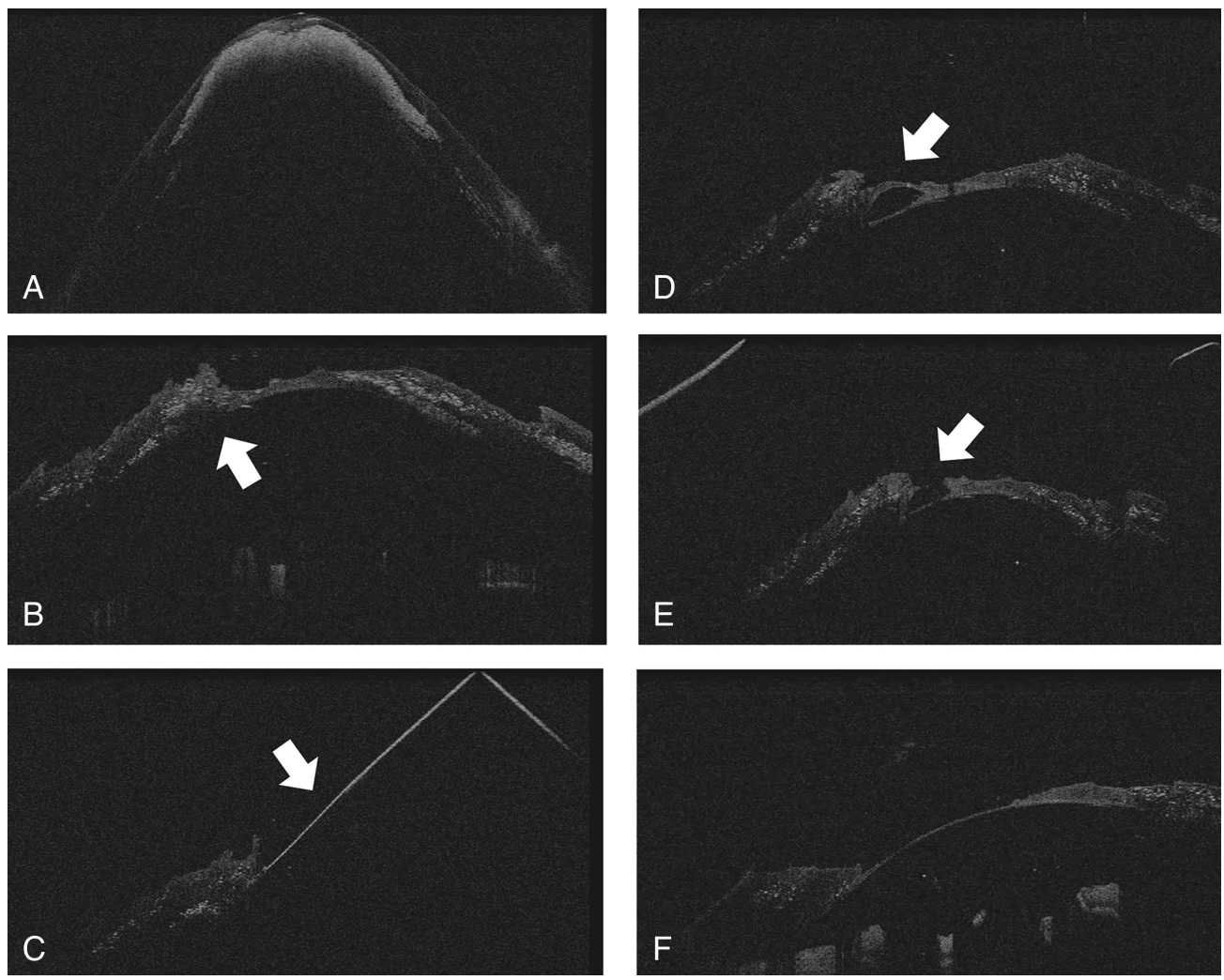

Figure 2 Incomplete big-bubble formation after air injection in deep anterior lamellar keratoplasty. Rescue attempt using the 'micro-bubble incision technique' (for details see ref. ${ }^{15}$ ). (A) Intrastromal air (white tissue) without big-bubble formation. After lamellar dissection (B), remaining intrastromal predescemetic air bubbles are sequentially incised using a sharp instrument $\left(15^{\circ} \mathrm{knife}\right.$, arrow; $\left.\mathrm{C}\right)$. That was repeated with more and deeper air bubbles ( $D$, arrow and $E$ ), which were then opened to expose bare Descemet's membrane (F).

steps of DALK to view the tissue from another angle without interrupting the procedure itself. Furthermore, by integrating the iOCT into the microscope and adapting its functions to zoom, focus and image acquisition, an online OCT imaging during surgery is enabled, as recently described to improve DMEK, that is, posterior lamellar corneal transplant surgery. ${ }^{10}$

In case of DALK, iOCT demonstrated to be a helpful device in verifying trephination depth and needle insertion in DALK for precise identification of the accurate preparation depth. Very low or extremely deep trephination depths would, therefore, be accurately observed prior to further preparation steps, for example, leading to retrephination, or particular attention, in needle placement. Although the use of a femtosecond laser reliably improves accuracy of the trephination depth, iOCT would assist in manual trephination, still used by the majority of corneal surgeons performing DALK. Preparation of bare DM becomes easier due to intraoperative control of remaining stromal tissue, however, DM rupture could not be prevented in all cases investigated in this small series. One reason could have been that in younger patients, corneal stroma adherence is increased in comparison to older patients requiring a higher manual force during dissection and, thereby, increasing the risk of perforation. Future technical developments, such as 3D real-time OCT imaging of the entire cornea would enable the surgeon to locate instruments without constantly aligning the OCT-beam which would then allow to better navigate and reduce complication rates in particularly difficult cases. In case of lacking big bubble formation, 'Microbubble incision technique' controlled by iOCT facilitated safer preparation of DM due to definite localisation of artificial intrastromal cavities. ${ }^{15}$ Furthermore, visualisation and drainage of clinically invisible interface fluid would avoid delayed healing and reduce air injection rates for this indication. Overall, the presented iOCT is the first intraoperative OCT device that is fully integrated into a surgical microscope, enabling real-time OCT imaging during surgical procedures of the
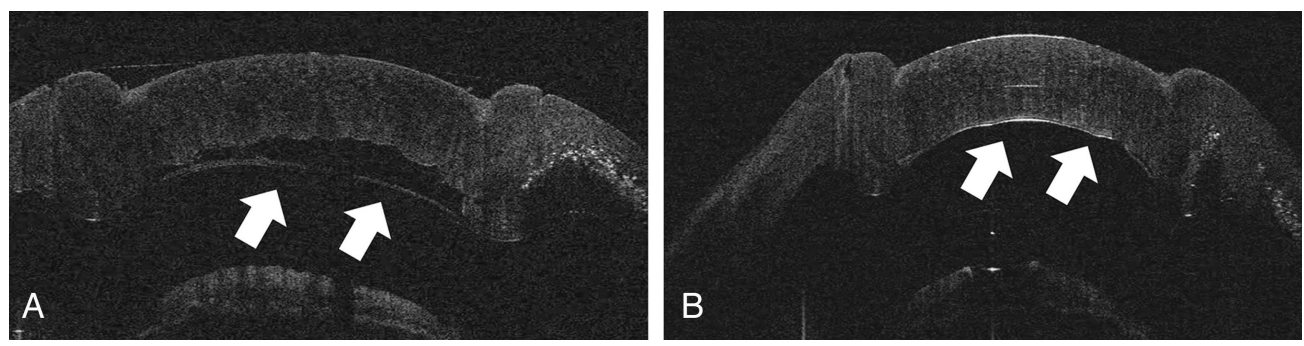

Figure 3 (A) Intraoperative optical coherence tomography enables to assure graft attachment to Descemet's membrane (arrows) in deep anterior lamellar keratoplasty (DALK) and guides drainage of interface fluid between donor stroma and host Descemet's membrane at the end of DALK surgery. Note absent fluid after drainage on the right side (B). 

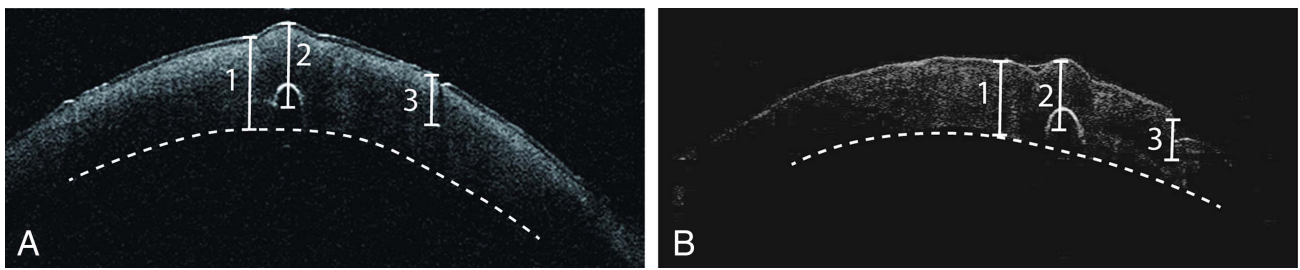

Figure 4 Quantitative measurements of corneal thickness, depth of the air injection needle and trephination depth (A) Representative paracentral optical coherence tomography (OCT) image of patient 5 with indicators for corneal thickness $(1=389 \mu \mathrm{m})$, depth of the air injection needle $(2=311 \mu \mathrm{m})$ and trephination depth $(3=270 \mu \mathrm{m})(B)$ Representative paracentral OCT image of patient 2 with indicators for corneal thickness $(1=333 \mu \mathrm{m})$, depth of the air injection needle $(2=296 \mu \mathrm{m})$ and trephination depth $(3=198 \mu \mathrm{m})$. The dotted line represents the posterior surface of the cornea.

eye. As shown for posterior lamellar keratoplasty (DMEK). ${ }^{10}$ iOCT also demonstrates benefits for anterior lamellar keratoplasty (DALK) and may aid surgeons, in particular, by steepening the learning curve at the beginning of surgical training in lamellar keratoplasty. In comparison with other imaging approaches, intraoperative OCT still has some limitations, such as higher costs, fixed angle and single scan direction with only limited degree of rotation. However, in challenging situations such as reduced visibility, uncertain tissue depth during lamellar preparation or failed big-bubble formation iOCT enables precise evaluation of the situation and possible 'rescue-measures' for successful completion of the procedure without the need of additional staff for handling hand-held devices and without the need to interrupt the procedure. Future developments such as 3D images of the entire surgical field and automated intraoperative depths measurements, together with larger prospective trials, will have to evaluate how much this technology can improve outcome measures in big-bubble DALK surgery.

Acknowledgements The authors would like to acknowledge the superb technical work of S Hackbarth and G Simons from the Cornea Bank, University Hospital of Cologne.

Contributors All authors have contributed either in the design or conduct of the study or performed the retrospective data analysis and writing of the manuscript.

Funding Deutsche Forschungsgemeinschaft (German Research Council: SFB 643 (B10), STE 1928/2-1, CU 47/4-1). Ruth und Helmut Lingen Stiftung, Köln. European Union FP7: COST BM 1302. Koeln Fortune Research Funding, Faculty of Medicine, University of Cologne. The sponsor or funding organisation had no role in the design or conduct of this research.

Competing interests $\mathrm{EL}$ and MK are employees of OptoMedical Technologies $\mathrm{GmbH}$, SO is employee of Moeller-Wedel GmbH, EL, MK and SO are shareholders of OptoMedical Technologies.

\section{Ethics approval}

Provenance and peer review Not commissioned; externally peer reviewed.

Data sharing statement Data will be available on request by the corresponding author.

Open Access This is an Open Access article distributed in accordance with the Creative Commons Attribution Non Commercial (CC BY-NC 3.0) license, which permits others to distribute, remix, adapt, build upon this work non-commercially, and license their derivative works on different terms, provided the original work is properly cited and the use is non-commercial. See: http://creativecommons.org/ licenses/by-nc/3.0/

\section{REFERENCES}

1 Anwar M, Teichmann KD. Big-bubble technique to bare Descemet's membrane in anterior lamellar keratoplasty. J Cataract Refract Surg 2002;28:398-403. Epub 2002/04/26
2 Cursiefen C, Heindl LM. [Perspectives of deep anterior lamellar keratoplasty]. Der Ophthalmologe: Zeitschrift der Deutschen Ophthalmologischen Gesellschaft 2011:108:833-9.

3 Shimmura S, Tsubota K. Deep anterior lamellar keratoplasty. Curr Opin Ophthalmol 2006:17:349-55

4 Steven $\mathrm{P}$, Hos D, Heindl LM, et al. [Immune reactions after DMEK, DSAEK and DALK]. Klinische Monatsblatter fur Augenheilkunde. 2013;230:494-9.

5 Fontana L, Parente $\mathrm{G}$, Sincich $\mathrm{A}$, et al. Influence of graft-host interface on the quality of vision after deep anterior lamellar keratoplasty in patients with keratoconus. Cornea. 2011;30:497-502.

6 Reinhart WJ, Musch DC, Jacobs DS, et al. Deep anterior lamellar keratoplasty as an alternative to penetrating keratoplasty a report by the american academy of ophthalmology. Ophthalmology 2011;118:209-18.

7 Mueller M, Steven P, Lankenau E, et al. Intraoperative OCT (IOCT) for Anterior and Posterior Segment Surgery. ARVO: IOVS; 2010.

8 Scorcia V, Busin M, Lucisano A, et al. Anterior segment optical coherence tomography-guided big-bubble technique. Ophthalmology 2013;120:471-6.

9 Wykoff CC, Berrocal AM, Schefler AC, et al. Intraoperative OCT of a full-thickness macular hole before and after internal limiting membrane peeling. Ophthalmic Surg Lasers Imaging 2010;41:7-11.

10 Steven $\mathrm{P}$, Le Blanc $\mathrm{C}$, Velten $\mathrm{K}$, et al. Optimizing descemet membrane endothelia keratoplasty using intraoperative optical coherence tomography. JAMA ophthalmology 2013;131:1135-42.

11 Lankenau E, Klinger D, Winter C, et al. Combining Optical Coherence Tomography (OCT) with an operating micoscope. In: Buzug TM, Holz D, Bongartz J, Kohl-Bareis M, Hartmann U, Weber S, eds. Advances in medical engineering. Berlin, Heidelberg, New York: Springer; 2007;343-8.

12 Heindl LM, Riss S, Bachmann BO, et al. Split cornea transplantation for 2 recipients: a new strategy to reduce corneal tissue cost and shortage. Ophthalmology 2011:118:294-301.

13 Heindl LM, Riss S, Laaser K, et al. Split cornea transplantation for 2 recipientsreview of the first 100 consecutive patients. Am JOphthalmol 2011; 152:523-32 e2.

14 Braun JM, Hofmann-Rummelt C, Schlotzer-Schrehardt U, et al. Histopathological changes after deep anterior lamellar keratoplasty using the 'big-bubble technique'. Acta Ophthalmol 2013:91:78-82.

15 Riss S, Heindl LM, Bachmann BO, et al. Microbubble incision as a new rescue technique for big-bubble deep anterior lamellar keratoplasty with failed bubble formation. Cornea. 2013:32:125-9.

16 Riss S, Heindl LM, Bachmann BO, et al. Pentacam-based big bubble deep anterior lamellar keratoplasty in patients with keratoconus. Cornea 2012; 31:627-32.

17 Huttmann G, Lankenau E, Schulz-Wackerbarth C, et al. [Optical coherence tomography: from retina imaging to intraoperative use-a review]. Klinische Monatsblatter fur Augenheilkunde 2009;226:958-64.

18 Yeh RY, Quilendrino R, Musa FU, et al. Predictive value of optical coherence tomography in graft attachment after Descemet's membrane endothelial keratoplasty. Ophthalmology. 2013;120:240-5

19 Steven $\mathrm{P}$, Heindl LM, Hos D, et al. Immune reactions after DMEK, DSAEK and DALK. Klin Monbl Augenheilkd 2013;230:494-9.

20 Lankenau E, Krug M, Oelckers $S$, et al. iOCT with surgical microscopes: a new imaging during microsurgery. Adv Opt Technol 2013:2:233-9.

21 Burkhart ZN, Feng MT, Price MO, et al. Handheld slit beam techniques to facilitate DMEK and DALK. Cornea. 2013:32:722-4. 